\title{
Intrinsic scatter of caustic masses and hydrostatic bias: An observational study
}

\author{
S. Andreon ${ }^{1}$, G. Trinchieri ${ }^{1}$, A. Moretti ${ }^{1}$, and J. Wang ${ }^{2}$ \\ 1 INAF-Osservatorio Astronomico di Brera, via Brera 28, 20121 Milano, Italy \\ e-mail: stefano. andreon@brera.inaf.it \\ 2 Dep. of Physics and Astronomy, University of the Western Cape, Cape Town 7535, South Africa
}

Received 3 April 2017 / Accepted 13 July 2017

\begin{abstract}
All estimates of cluster mass have some intrinsic scatter and perhaps some bias with true mass even in the absence of measurement errors for example caused by cluster triaxiality and large scale structure. Knowledge of the bias and scatter values is fundamental for both cluster cosmology and astrophysics. In this paper we show that the intrinsic scatter of a mass proxy can be constrained by measurements of the gas fraction because masses with higher values of intrinsic scatter with true mass produce more scattered gas fractions. Moreover, the relative bias of two mass estimates can be constrained by comparing the mean gas fraction at the same (nominal) cluster mass. Our observational study addresses the scatter between caustic (i.e., dynamically estimated) and true masses, and the relative bias of caustic and hydrostatic masses. For these purposes, we used the X-ray Unbiased Cluster Sample, a cluster sample selected independently from the intracluster medium content with reliable masses: 34 galaxy clusters in the nearby $(0.050<$ $z<0.135)$ Universe, mostly with $14<\log M_{500} / M_{\odot} \lesssim 14.5$, and with caustic masses. We found a $35 \%$ scatter between caustic and true masses. Furthermore, we found that the relative bias between caustic and hydrostatic masses is small, $0.06 \pm 0.05$ dex, improving upon past measurements. The small scatter found confirms our previous measurements of a highly variable amount of feedback from cluster to cluster, which is the cause of the observed large variety of core-excised X-ray luminosities and gas masses.
\end{abstract}

Key words. galaxies: clusters: general - galaxies: clusters: intracluster medium - X-rays: galaxies: clusters - methods: statistical

\section{Introduction}

Clusters of galaxies are the largest collapsed objects in the hierarchy of cosmic structures (e.g., Sarazin 1988). They arise from the smooth sea of hot particles and light under the action of gravity modulated by the action of dark energy and dark matter (e.g., Dressler et al. 1996; Weinberg et al. 2013). Our understanding of the gravitational processes that shape the cosmic web, which allow us to use galaxy clusters as cosmological probes (e.g., Vikhlinin et al. 2009), and of the interplay between dark matter and baryonic components (e.g., Young et al. 2011, and references therein), relies on scaling relations between halo mass and observable quantities tracing one of their constituting and observable parts, such as galaxies, intracluster medium, or dark matter. All of the methods to weight galaxy clusters using these observables are subject to biases due to scatter between the mass and observable or its dependency on other physical cluster properties. Even the direct observation of the total matter, via weak lensing, is subject to scatter with mass due to cluster triaxiality, large scale structure, and intrinsic alignments (Meneghetti et al. 2010; Becker \& Kravtsov 2011).

The caustic technique derives masses from measurements of the line-of-sight escape velocity. Caustic masses are unaffected by the dynamical state of the cluster and by large scale structure (Diaferio 1999; Serra et al. 2011), however they are affected by elongation along the line of sight. Previous analyses found that caustic masses have low scatter with true mass, but the results are based on simulations, or are indirect or noisy. In fact, numerical simulations (Serra et al. 2011; Gifford \& Miller 2013) showed a
$20 \%$ scatter, Geller et al. (2013) showed that weak lensing and caustic masses agree within 30\%, and Maughan et al. (2016) found a $23 \pm 11 \%$ scatter with hydrostatic masses. Andreon \& Congdon (2014) found a small $(\ll 0.1 \mathrm{dex})$ intrinsic scatter between richness and weak lensing mass, while Andreon (2012) found a small ( $\ll 0.1$ dex) scatter between richness and caustic masses. Although indirect, the points above suggest that a large scatter between caustic and true mass is unlikely, given the small scatter with weak lensing masses, hydrostatic masses, and richness.

The first aim of this paper is a data-driven determination of the intrinsic scatter of caustic masses. We achieve this objective by an innovative approach which can be applied to other types of masses as well. We exploit the fact that a large scatter in halo mass induces a large scatter on gas fraction (cosmic conspiracy notwithstanding); the latter is proportional to one over halo mass. Therefore, a value of scatter of the gas fraction can be converted into an upper limit of the intrinsic scatter of the caustic masses as detailed in Sect. 3. If caustic masses were low scatter proxies of true masses, then they could be useful to calibrate noisier mass proxies. They could also be used to measure mass-related cluster properties free of the large scatter of weak lensing masses due to triaxiality, large scale structure, and intrinsic alignment.

A second aim is to measure the relative bias of caustic and hydrostatic masses. As mentioned, different methods to estimate galaxy cluster masses may also return systematically underestimated, or overestimated masses. Hydrostatic masses, i.e., masses derived under the assumption of hydrostatic equilibrium, have been often used both to measure intracluster medium properties 
(e.g., Vikhlinin et al. 2006; Arnaud et al. 2007) and to calibrate other mass proxies such as the integrated pressure (e.g., Arnaud et al. 2010) and integrated pseudo-pressure (Arnaud et al. 2007; Vikhlinin et al. 2009). Hydrostatic masses are known to be slightly biased estimates of true mass because of deviations from the hydrostatic equilibrium or the presence of nonthermal pressure support such as turbulence, bulk flows, or cosmic rays (e.g., Rasia et al. 2006; Nagai et al. 2007; Nelson et al. 2014). The amount of the hydrostatic bias is uncertain, but usually estimated at 10 to $20 \%$. However, a much larger bias has been invoked to reconcile cosmological parameters derived from the cosmic microwave background and cluster counts (Planck Collaboration XX 2014), although the calibration of the bias by Planck team has been amply discussed (von der Linden 2014; Andreon 2014; Smith et al. 2016). Caustic masses can provide an alternative calibration of the bias of hydrostatic masses. They are almost unused for this purpose (we are only aware of Maughan et al. 2016) and we exploit a new method for using this type of masses: if there is a relative bias between caustic and hydrostatic masses then there should be an offset in gas mass derived using the two masses.

Throughout this paper, we assume $\Omega_{\mathrm{M}}=0.3, \Omega_{\Lambda}=0.7$, and $H_{0}=70 \mathrm{~km} \mathrm{~s}^{-1} \mathrm{Mpc}^{-1}$. Results of stochastic computations are given in the form $x \pm y$, where $x$ and $y$ are the posterior mean and standard deviation. The latter also corresponds to $68 \%$ intervals because we only summarize posteriors close to Gaussian in this way. Logarithms are in base 10 .

\section{Sample selection, cluster masses, and X-ray data}

Sample selection, halo mass derivation, and X-ray data are presented and discussed in Andreon et al. (2016, Paper I), and gas masses are derived in Andreon et al. (2017, Paper II), to which we refer for details. We summarize here the work done. We used a sample of 34 clusters in the very nearby universe $(0.050<z<0.135$, XUCS, for X-ray Unbiased Cluster Sample) extracted from the $\mathrm{C} 4$ catalog (Miller et al. 2005) in regions of low Galactic absorption. There is no X-ray selection in our sample, meaning that 1) the probability of inclusion of the cluster in the sample is independent of its X-ray luminosity (or count rate), and 2) no cluster is kept or removed on the basis of its $\mathrm{X}$-ray properties, except for two clusters for which we cannot derive gas masses. The impact of this selection is discussed in Sect. 3.3.

We collected the few X-ray observations present in the $X M M$-Newton or Chandra archives and we observed the remaining clusters with $S$ wift (individual exposure times between 9 and $31 \mathrm{ks}$ ), as detailed in Table 1 of Paper I. Swift observations have the advantage of a low X-ray background (Moretti et al. 2009), making it extremely useful for sampling a cluster population that includes low surface brightness clusters (Andreon \& Moretti 2011).

Caustic masses within $r_{200}{ }^{1}, M_{200}$, have been derived following Diaferio \& Geller (1997), Diaferio (1999), and Serra et al. (2011), then converted into $r_{500}$ and $M_{500}$ assuming a Navarro et al. (1997) profile with concentration $c=5$. Adopting $c=3$ would change mass estimates by a negligible amount; see Paper I. The median number of members within the caustics is 116 and the interquartile range is 45 . The median mass of the cluster sample, $\log M_{500} / M_{\odot}$, is 14.2 and the interquartile range is 0.4 dex. The average mass error is 0.14 dex.

\footnotetext{
1 The radius $r_{\Delta}$ is the radius within which the enclosed average mass density is $\Delta$ times the critical density at the cluster redshift.
}

Gas masses are derived by projecting a flexible radial profile, fitting its projection to the unbinned X-ray data, and propagating all modeled sources of uncertainties (e.g., spectral normalization, variation in exposure time including those originated by vignetting or excised regions) with their non-Gaussian behavior (when relevant) into the gas mass estimate using Bayesian methods. The spectral normalization, measured in the annulus $0.15<r / r_{500}<0.5$, is used to convert brightnesses in gas densities. The average gas mass error is $0.10 \mathrm{dex}$, as detailed and extensively tested, in Paper II.

\section{Analysis and results}

In Paper II, we fitted the relation between gas fraction and mass, allowing an intrinsic scatter in $f_{\text {gas }} \mid M$, while freezing the scatter between caustic and true masses to 0.08 dex. In this paper, we let it free to vary.

In detail, we allow caustic masses to have an additional scatter against true halo masses $\sigma_{\text {intr. caus. to be added to our errors }}$ on $\log M^{\text {obs }}$, which already include a $20 \%$ intrinsic scatter between caustic masses and true masses (already accounted for in $\left.\sigma_{\log M, i}\right)$

$\log M_{i}^{\text {obs }} \sim N\left(\log M_{i}, \sigma_{\log M, i}^{2}+\sigma_{\text {addit.scat. }}^{2}\right)$.

We use a linear model with intrinsic scatter $\sigma_{\text {intr }}$ in $(\log )$ gas fractions,

$\log M_{i, \mathrm{gas}} \sim N\left(a\left(\log M_{i}-14\right)+\log M_{\mathrm{gas}, 14}, \sigma_{\mathrm{intr}}^{2}\right)$.

We fit the data in the gas mass versus halo mass plane, where errors are less correlated (see Andreon 2010), i.e.,

$\log M_{i, \text { gas }}^{\text {obs }} \sim N\left(\log M_{i, \mathrm{gas}}, \sigma_{\log M_{\mathrm{gas}}, i}^{2}\right)$.

Since we cannot properly determine the slope of the relation in the limited range covered by XUCS clusters, we adopt as prior the posterior derived in Andreon (2010) for the sample in Vikhlinin et al. (2006) and Sun et al. (2009), $0.15 \pm 0.03$ as follows:

$a \sim N\left(1+0.15,0.03^{2}\right)$.

As shown in Sect. 3.3, the assumption of the slope value does not change the results because the clusters studied have similar masses. For the remaining parameters (namely: additional caustic scatter, intrinsic scatter, and $\log$ of gas fraction at $\log M / M_{\odot}=$ $\left.14, \log f_{\text {gas }, 14}=\log M_{\text {gas }, 14}-14\right)$, we assume a uniform and wide range of values that includes the true value as follows:

$\log M_{i} \sim U(13.3,15.5)$

$\sigma_{\text {intr }} \sim U(0.01,1)$

$\sigma_{\text {addit.scat. }} \sim U(0,3)$

$\log M_{\text {gas }, 14} \sim U(10,14)$.

The parameter space is sampled by Gibbs sampling using JAGS (see Andreon 2011). For the three clusters for which multiple estimates for gas fraction are available, derived from different telescopes, the fit only uses those with smaller errors.

As detailed in Paper II, knowledge of the selection function in the observable and its covariance with the studied quantity is in general essential to propagate selection effects from the quantity used to select the sample to the quantity of interest (here gas mass). The use of an X-ray unbiased cluster sample, such that used in our paper, does not require the application of any correction for the selection function to fit (often difficult to apply). 


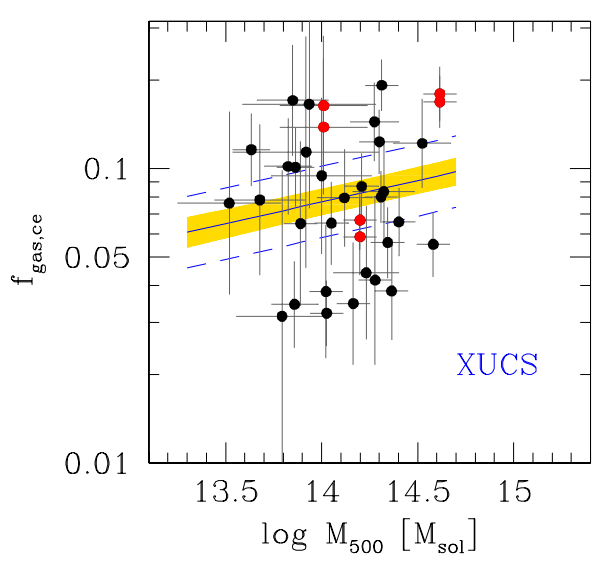

Fig. 1. Core-excised gas fraction $f_{\text {gas,ce }}$ vs. halo mass $M_{500}$ of XUCS sample. The solid line indicates the mean relations, the shading indicates its $68 \%$ uncertainty, whereas the dashed lines indicate the mean relation $\pm 1 \sigma_{\text {intr }}$. Close pairs of red points (with identical masses) indicate measurements from different X-ray telescopes.

\section{Using core excised gas masses, we found}

$\log f_{\text {gas }}=(0.15 \pm 0.03)\left(\log M_{500}-14\right)-1.11 \pm 0.04$,

with the slope posterior largely determined by the slope prior.

The results of the fit are plotted in Fig. 1, including the mean relation (solid line), its $68 \%$ uncertainty (shading), and the mean relation $\pm 1 \sigma_{\text {intr }}$.

\subsection{Constraints on the scatter of caustic masses from the gas fraction scatter}

The top left panel of Fig. 2 shows the joint posterior probabilities distributions of intrinsic and additional scatters. The scatter of the data sets a joint constraint on the intrinsic scatter and on the additional caustic scatter (approximatively on their sum). In particular, if $\sigma_{\text {intr }} \gtrsim 0.15$, the most probable additional scatter is $<0.1$, i.e., caustic masses have very little additional scatter with true mass above what has already been considered in the mass error $(0.08 \mathrm{dex})$. If $\sigma_{\text {intr }} \approx 0$ then the most probable value of additional scatter is $\sim 0.15$ dex, i.e., scatter with true mass is underestimated by $40 \%$ at most. For whatever (positive) intrinsic scatter, the additional caustic scatter cannot exceed 0.3 dex (bottom left panel) because higher values require a data scatter that is larger than the one observed. Finally, marginalizing (averaging) over all possible values, the additional caustic scatter is less than 0.19 dex with $95 \%$ probability (see bottom left panel), which brings the posterior mean of the total scatter to 0.13 dex and the $95 \%$ upper limit of the total scatter of caustic masses to 0.21 dex. This observation-based $35 \%$ scatter confirms and improves upon other indirect or noisy evidence (see introduction) and makes caustic masses the prime choice to measure mass, in particular free of the biases of other mass estimates (such as hydrostatic masses).

The idea of exploiting the observed scatter in the gas fraction to derive an upper limit to the scatter of a mass estimate can be applied to other mass estimates, such as hydrostatic or weak lensing masses. However, although the idea is promising, the application to other samples needs to carefully account for complications which are absent in our X-ray Unbiased Cluster Sample but present in other samples such as: a) in samples selected using their content in gas mass (or a quantity showing covariance with it, such as X-ray luminosity; see Paper II);
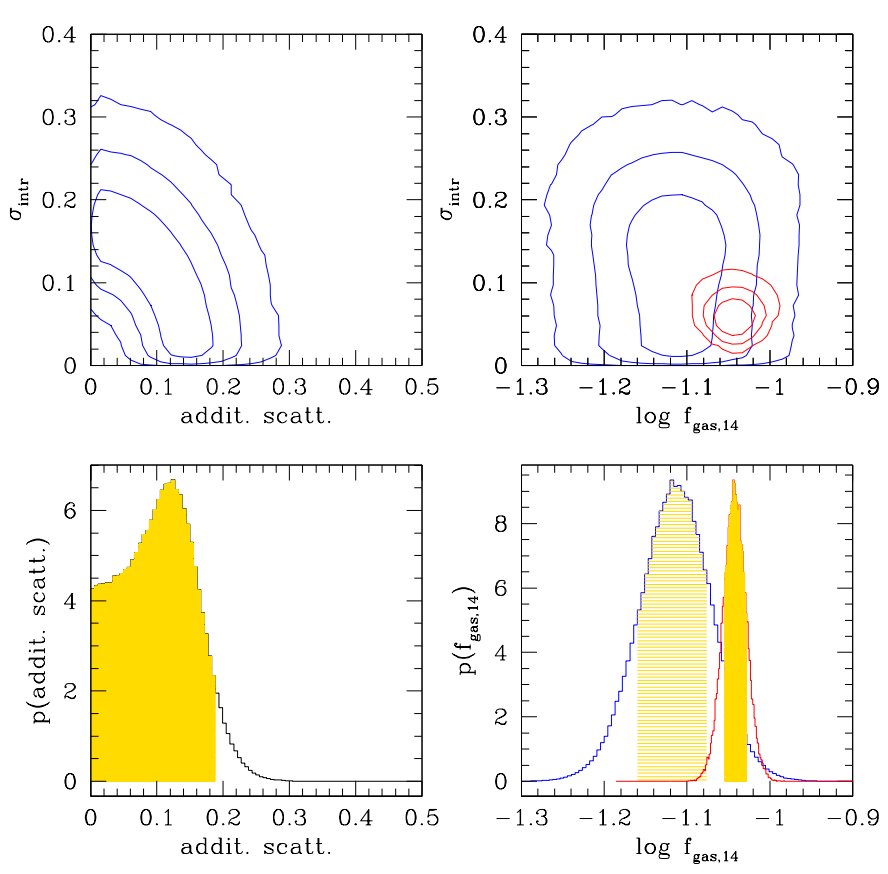

Fig. 2. Joint $68 \%, 95 \%$, and $99.7 \%$ (highest posterior) probability contours (top panels) of our fit to XUCS cluster (in blue) and to an X-ray selected sample of relaxed clusters (in red, from Andreon 2010). The bottom panels show the marginals: the posterior probability distribution of the gas fraction (bottom right panel) and the additional scatter of caustic masses (bottom left panel). The shading indicates the $68 \%$ (highest posterior) probability in the bottom right panel and the $95 \%$ probability in the bottom-left panel.

b) in samples including only a cluster subpopulation; c) in samples selected with unknown or ill-defined selection function; or d) in samples in which the reference radius used to determine the gas fraction depends on the X-ray data (as in hydrostatic mass estimates).

\subsection{The small relative bias of hydrostatic and caustic masses}

In this section we set a limit to the relative bias of hydrostatic and caustic masses by comparing the average gas fraction of clusters with $\log M / M_{\odot}=14$, as derived using caustic and hydrostatic masses. If a bias exists, then it should appear as a difference in the average gas fraction of clusters with the same nominal halo mass. The compared fractions are based on XUCS for clusters with caustic masses and on Vikhlinin et al. (2006) and Sun et al. (2009) for clusters with hydrostatic masses.

As mentioned in the introduction, a large bias has been invoked to reconcile cosmological parameters derived by the Planck team from different probes. Figure 3 illustrates the variety of the hydrostatic biases $1-b$ found; the lefmost solid line is the bias needed to reconcile cosmological parameters (Planck Collaboration XXIV 2016) and the other curves show independently derived mass biases (von der Linden et al. 2014; Hoekstra et al. 2015; Smith et al. 2016), which are often called mass priors in the literature. As shown in the figure, there is some tension between the various determinations. However, the selection function is either not available or not accounted for in the computation of the bias (except for the black curve), which may affect the derived bias (see Andreon 2016).

The core-excised gas fraction vs. halo mass mean relation of XUCS has been derived in Sect. 3.1, while the non-core-excised 


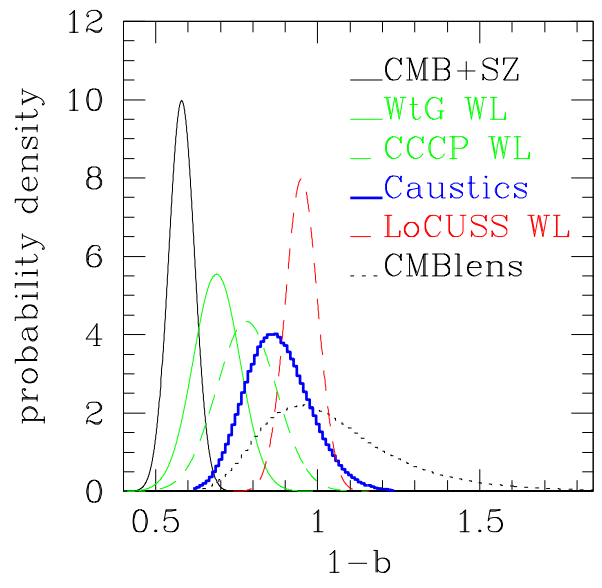

Fig. 3. Comparison of estimates of the hydrostatic bias $1-b$. The leftmost (solid black) curve is the distribution needed to reconcile cluster counts and cosmological constraint from CMB (Planck Collaboration XXIV 2016). The dotted broad distribution reaching $1-b=1.5$ is the bias found using lensing of CMB temperature anisotropies (Planck Collaboration XXIV 2016). Other curves refer to weak lensing mass estimates (von der Linden et al. 2014; Hoekstra et al. 2015; Smith et al. 2016) or our work (blue thick curve centered on $1-b=0.88 \approx$ $1-0.06 \ln 10$ ). All probability densities integrate to one (as probability axioms prescribe) and we converted without approximations the distribution in $1 /(1-b)$ (from lensing from $\mathrm{CMB}$ ) to the plotted $1-b$ distribution.

gas fractions were used for clusters in Vikhlinin et al. (2006) and Sun et al. (2009) and the relation was derived in Andreon (2010). The bottom right and top right panels of Fig. 2 show the posterior distribution and joint probability contours for both samples. The gas fractions of the two samples at $\log M / M_{\odot}=14$ only differ by $0.06 \pm 0.05$ dex, where XUCS gas fraction is lower, after accounting for the negligible $(0.01 \mathrm{dex})$ difference between core-excised and non-core excised gas masses for XUCS clusters (derived in Paper II). A similar conclusion may be qualitatively derived from Fig. 4 of Paper II, where we plotted the fit on the Vikhlinin et al. (2006) and Sun et al. (2009) sample on XUCS individual values.

The close agreement of the two gas fractions may have two origins. First, there is a negligible relative bias $(0.06 \pm 0.05$ dex $)$ between caustic and hydrostatic mass scales (see Fig. 3). Our result is roughly consistent with that of Maughan et al. (2016) $(-0.08 \pm 0.04)$. Second, the agreement is the result of a fine tuning between an hydrostatic mass bias and sample selection. The comparison sample, drawn from clusters in Vikhlinin et al. (2006) and Sun et al. (2009), is formed by clusters selected to be relaxed but has otherwise an unknown representativeness because the sample has an unknown selection function. Nevertheless, these clusters are those used to calibrate the observablemass relations used by Vikhlinin et al. (2009) to constrain cosmological parameters, which are found to be in agreement with those based on other probes, suggesting that these clusters do not provide biased scaling relations. To have the small offset between the derived gas fraction as result of selection effects compensating real differences, one of the two following conditions are requested. First, hydrostatic masses would be underestimated and gas-rich (and photon-rich) would be clusters preferentially discarded in Vikhlinin et al. (2006) and Sun et al. (2009). However, there is no reason why gas-rich clusters should be preferentially discarded. Second, hydrostatic masses would be overestimated, but there is no evidence in the literature for that. These make fine tuning an unlikely possibility.
We therefore conclude that a relative bias between caustic and hydrostatic masses is small, if it exists at all, at least in the redshift and mass ranges explored by our data, i.e., for clusters in the nearby $(0.050<z<0.135)$ Universe, mostly with $14<\log M_{500} / M_{\odot} \lesssim 14.5$. If the result can be extrapolated to slightly more massive clusters at intermediate distances (i.e., to the Planck cosmological sample), then the source of the tension between cluster counts and CMB cosmological parameters should be looked for somewhere else, for example invoking a possible non-self-similar evolution of cluster scaling relations (Andreon 2014) or a common bias for caustic and hydrostatic masses.

\subsection{Sensitivity analysis}

Our starting sample is formed by 34 clusters that form an X-ray unbiased sample. However, two of these clusters are discarded in the course of the analysis because of their X-ray properties. We verified whether this alters the original properties of the sample. We checked that the intercept of Eq. (1) changes by less than 0.01 dex if we reintroduce the two clusters using a gas mass predicted from $L_{X}$ (Paper II), which implies that our conclusion on the relative bias of hydrostatic and caustic masses (Sect. 3.2) is unaffected.

Two objects are an unsufficient number to alter the scatter of the whole sample (34 objects) population and therefore our constraint on the scatter of caustic masses from the gas fraction scatter is robust.

Finally, our analysis of the gas fraction versus mass assumed as slope prior the posterior of Andreon (2010) based on Vikhlinin et al. (2006) and Sun et al. (2009) clusters because of the limited range in mass covered by XUCS clusters. If we instead took a uniform prior on the angle in order to allow different slopes, we found identical scatters and intercept and also joint posterior distributions of the key parameters (gas fraction, intrinsic scatter, and additional scatter of caustic masses) close to those depicted in Fig. 2. This shows the robustness of our conclusions on assumptions about the slope.

\subsection{Revisiting our previous papers}

In Paper I we kept the intrinsic scatter between true and caustic massed fixed at the value of 0.08 dex and we found a 0.5 dex scatter in $L_{\mathrm{X}} \mid M_{\mathrm{c}}$, which is a surprising large value not seen before. Smaller scatters were probably the result of the selection of the samples through the ICM content. Paper I assumed, however, that caustic masses have a small scatter with true mass. In the current paper we show that our assumption was correct and therefore our interpretation was correct. Quantitatively, to induce a 0.5 dex scatter in X-ray luminosity at a given mass, caustic masses should have a $\sigma\left(M_{\mathrm{c}} \mid M_{\mathrm{t}}\right) \sim 0.6$ dex scatter, that is ruled out by a large margin by the scatter in the gas fraction (Sect. 3.2). Therefore, the big variance in core-excised X-ray luminosities found in Paper I is real and not induced by an unaccounted scatter between true and caustic masses.

In Paper II, we fitted gas fraction as we do in this paper, but freezing the scatter between true and caustic masses at the value of 0.08 dex. The intercept we derive here allowing caustic masses to have a free amount of intrinsic scatter is in full agreement with that derived in Paper II. The intrinsic scatter in gas fraction derived in the present paper depends on the prior on the intrinsic scatter adopted for caustic masses 
because, as mentioned above, the data only offer a constraint on the combination of the two. The value of intrinsic scatter found here, $0.12 \pm 0.06 \mathrm{dex}$, is marginally lower than found in Paper II, $0.17 \pm 0.04$ because the difference has been attributed to the scatter between real and caustic masses. Although lower (by a statistical insignificant difference), the current scatter confirms our conclusion in Paper II of a highly variable, cluster-to-cluster, amount of gas within $r_{500}$. This indicates that the large scatter in gas fraction seen in Paper II is not due to an unaccounted for scatter in caustic masses. Instead, it is due to a highly variable, cluster-to-cluster, amount of gas within $r_{500}$. These differences are much larger than found in the literature for subsamples of the whole cluster population.

To summarize, the stringent upper limit to the scatter between caustic and true masses confirms that results in Papers I and II are not due to our assumption of a small scatter between true and caustic masses.

\section{Conclusions}

In this paper we introduced an innovative approch to derive an upper limit to the scatter between mass and proxy and to estimate the relative bias of two mass proxies. We applied it to caustic masses, and we note that it can be applied to other types of masses.

We used a sample of 34 clusters with caustic masses that we observed in X-ray and whose selection is, at a given cluster mass, independent of the intracluster medium content (see Paper I). In Paper II we derived gas masses by projecting a flexible radial profile and fitting its projection to the unbinned X-ray data. We also fit gas mass versus halo mass while keeping the intrinsic scatter between true and caustic massed fixed at the value of $0.08 \mathrm{dex}$. In this paper we modify the fitting model to include an additional source of scatter, i.e. the scatter between true and caustic masses. We then exploit the fact that the observed scatter in the gas fraction is inflated by halo mass errors, and therefore an estimate of the former sets an upper limit on the scatter of caustic mass estimates. We found a $35 \%$ scatter between caustic and true masses.

Then, we set a limit to the relative bias between hydrostatic and caustic masses by comparing the average gas fraction of clusters with $\log M / M_{\odot}=14$ derived using caustic and hydrostatic masses. We found a small, if any, difference, $0.07 \pm 0.05 \mathrm{dex}$, with caustic masses being larger and with the caveat that, as other works in literature, the sample (part of it, in our case) has an unknown representativeness. This amount of bias is, by a large margin, too small to reconcile cosmological parameters derived by the Planck team from different probes.
The small scatter measured between caustic and true masses confirms our results in previous papers, which assumed the scatter to be small. We therefore confirm that the overall gas fraction is different from cluster to cluster, indicating a variable amount of feedback from cluster to cluster. By ruling out a large scatter between true and caustic masses we also confirm that the large variety of core-excised X-ray luminosities observed in Paper I is due to scatter into the amount of gas, rather than to the gas mass distribution within $r_{500}$.

\section{References}

Andreon, S. 2010, MNRAS, 407, 263

Andreon, S. 2011, in Astrostatistical Challenges for the New Astronomy, ed. J. Hilbe, Springer Series on Astrostatistics (Springer)

Andreon, S. 2012, A\&A, 548, A83

Andreon, S. 2014, A\&A, 570, L10

Andreon, S. 2016, A\&A, 587, A158

Andreon, S., \& Congdon, P. 2014, A\&A, 568, A23

Andreon, S., \& Moretti, A. 2011, A\&A, 536, A37

Andreon, S., Serra, A. L., Moretti, A., \& Trinchieri, G. 2016, A\&A, 585, A147 (Paper I)

Andreon, S., Wang, J., Trinchieri, G., Moretti, A., \& Serra, A. L. 2017, A\&A, 606, A24 (Paper II)

Arnaud, M., Pointecouteau, E., \& Pratt, G. W. 2007, A\&A, 474, L37

Arnaud, M., Pratt, G. W., Piffaretti, R., et al. 2010, A\&A, 517, A92

Becker, M. R., \& Kravtsov, A. V. 2011, ApJ, 740, 25

Diaferio, A. 1999, MNRAS, 309, 610

Diaferio, A., \& Geller, M. J. 1997, ApJ, 481, 633

Dressler, A., \& HST and Beyond Committee 1996, Exploration and the Search for Origins: A Vision for Ultraviolet- Optical-Infrared Space Astronomy (Washington, D.C.: Association of Universities for Research in Astronomy) Geller, M. J., Diaferio, A., Rines, K. J., \& Serra, A. L. 2013, ApJ, 764, 58 Gifford, D., \& Miller, C. J. 2013, ApJ, 768, L32

Hoekstra, H., Herbonnet, R., Muzzin, A., et al. 2015, MNRAS, 449, 685

Kravtsov, A. V., Vikhlinin, A., \& Nagai, D. 2006, ApJ, 650, 128

Maughan, B. J., Giles, P. A., Rines, K. J., et al. 2016, MNRAS, 461, 4182

Meneghetti, M., Rasia, E., Merten, J., et al. 2010, A\&A, 514, A93

Miller, C. J., Nichol, R. C., Reichart, D., et al. 2005, AJ, 130, 968

Moretti, A., Pagani, C., Cusumano, G., et al. 2009, A\&A, 493, 501

Nagai, D., Kravtsov, A. V., \& Vikhlinin, A. 2007, ApJ, 668, 1

Navarro, J. F., Frenk, C. S., \& White, S. D. M. 1997, ApJ, 490, 493

Nelson, K., Lau, E. T., Nagai, D., Rudd, D. H., \& Yu, L. 2014, ApJ, 782, 107

Planck Collaboration XX. 2014, A\&A, 571, A20

Planck Collaboration XXIV. 2016, A\&A, 594, A24

Rasia, E., Ettori, S., Moscardini, L., et al. 2006, MNRAS, 369, 2013

Sarazin, C. L. 1988, X-ray emission from clusters of galaxies (Cambridge: Cambridge University Press)

Serra, A. L., Diaferio, A., Murante, G., \& Borgani, S. 2011, MNRAS, 412, 800

Smith, G. P., Mazzotta, P., Okabe, N., et al. 2016, MNRAS, 456, L74

Sun, M., Voit, G. M., Donahue, M., et al. 2009, ApJ, 693, 1142

Vikhlinin, A., Kravtsov, A., Forman, W., et al. 2006, ApJ, 640, 691

Vikhlinin, A., Burenin, R. A., Ebeling, H., et al. 2009, ApJ, 692, 1033

von der Linden, A., Mantz, A., Allen, S. W., et al. 2014, MNRAS, 443, 1973

Young, O. E., Thomas, P. A., Short, C. J., \& Pearce, F. 2011, MNRAS, 413, 691

Weinberg, D., Mortonson, M. J., Eisenstein, D. J. et al. 2013, Phys. Rep., 530, 87 